\title{
THE MEAN VALUE OF THE ARTIN L-SERIES AND ITS DERIVATIVE OF A CUBIC FIELD
}

\section{by LENARD WEINSTEIN}

(Received 29 June, 1978)

1. Introduction. Let $K$ be a non-abelian cubic field of discriminant $D$, and $\zeta_{K}(s)$ its Dedekind zeta-function. Set $\psi(s)=\zeta_{K}(s) / \zeta(s)$. Then it is known that $\psi(s)$ is the Artin L-series associated with the field $K$. It is also known that $\psi(s)$ is an entire function of order 1.

If $K$ is not a totally real field then $\psi(s)$ satisfies the functional equation

$$
\psi(1-s)=\frac{2}{\sqrt{D}}\left(\frac{\sqrt{D}}{2 \pi}\right)^{2 s} \sin \pi s \Gamma^{2}(s) \psi(s) .
$$

If $K$ is a totally real field then $\psi(s)$ satisfies the functional equation

$$
\psi(1-s)=\frac{4}{\sqrt{D}}\left(\frac{\sqrt{D}}{2 \pi}\right)^{2 s} \cos ^{2} \frac{1}{2} \pi s \Gamma^{2}(s) \psi(s)
$$

Barrucand, in [1], has given asymptotic formulae for certain coefficient sums of $\psi(s)$. Here, using these results, and the methods of [2], [5] we prove the following:

THEOREM 1.

$$
\int_{0}^{\infty}\left|\psi\left(\frac{1}{2}+i t\right)\right|^{2} e^{-\delta t} d t=\frac{2 A}{\delta} \log \frac{1}{\delta}+O\left(\frac{1}{\delta}\right)
$$

for sufficiently small $\delta>0$.

(The positive constant $A=\frac{6 \mathrm{Ld}_{3}(1) \psi(1) D(2) E(2)}{\pi^{2} D(1) E(1)}$ is defined in [1, p. 962-A].)

Corollary 1.

$$
\int_{0}^{T}\left|\psi\left(\frac{1}{2}+i t\right)\right|^{2} d t \sim 2 A T \log T
$$

THEOREM 2.

$$
\int_{0}^{\infty}\left|\psi^{\prime}\left(\frac{1}{2}+i t\right)\right|^{2} e^{-\delta t} d t=\frac{8 A}{3 \delta} \log ^{3} \frac{1}{\delta}+O\left(\frac{1}{\delta} \log ^{2} \frac{1}{\delta}\right)
$$

for sufficiently small $\delta>0$.

Corollary 2.

$$
\int_{0}^{T}\left|\psi^{\prime}\left(\frac{1}{2}+i t\right)\right|^{2} d t \sim \frac{8}{3} A T \log ^{3} T
$$

Glasgow Math. J. 21 (1980) 9-18. 


\section{Lemmas.}

LeMma 1 (Van der Corput [4, p. 61]). Let $F(x)$ and $G(x)$ be real functions, $G(x) / F^{\prime}(x)$ monotonic and $F^{\prime}(x) / G(x) \geq m>0$, or $F^{\prime}(x) / G(x) \leq-m<0$, throughout the interval $(a, b)$. Then

$$
\left|\int_{a}^{b} G(x) e^{i F(x)} d x\right| \leq \frac{4}{m} .
$$

Lemma 2 (Euler Summation [4, p. 13]). Let $\phi(x)$ be a real function with a continuous derivative in the interval $(a, b)$. If, for $a \leq x \leq b, \phi^{\prime}(x) \geq 0$ or $\phi^{\prime}(x) \leq 0$, then

$$
\sum_{a \leq n \leq b} \phi(n)=\int_{a}^{b} \phi(x) d x+O(|\phi(a)|+|\phi(b)|) .
$$

The proof of the following lemmas follows easily from [1] and [2, p. 124], and will be omitted.

Lemma 3. Let $\psi(s)=\sum_{n=1}^{\infty} a(n) n^{-s} \quad(\sigma>1)$. Then

$$
\sum_{n \leq x} \frac{a^{2}(n)}{n}=A \log x+O(1)
$$

and

$$
\sum_{n \leq x} \frac{a^{2}(n) \log n}{n}=\frac{1}{2} A \log ^{2} x+O(\log x) .
$$

(The constant $A$ has been defined previously.)

LEMMA 4. Let $\psi^{\prime}(s)=\sum_{n=1}^{\infty} b(n) n^{-s}(\sigma>1)$, (Thus $b(n)=-a(n) \log n$.) Then, for sufficiently small $\beta>0$, we have

$$
\begin{gathered}
\sum_{n=1}^{\infty} \frac{a^{2}(n)}{n} e^{-n \beta}=A \log \frac{1}{\beta}+O(1), \\
\sum_{n=1}^{\infty} \frac{b^{2}(n)}{n} e^{-n \beta}=\frac{1}{3} A \log ^{3} \frac{1}{\beta}+O\left(\log ^{2} \frac{1}{\beta}\right)
\end{gathered}
$$

and

$$
\sum_{n=1}^{\infty} \frac{a^{2}(n) \log n}{n} e^{-n \beta}=O\left(\log ^{2} \frac{1}{\beta}\right)
$$

LEMMA 5.

$$
\sum_{n=1}^{\infty} \frac{\log ^{2} n}{n} e^{-n \beta}=\frac{1}{3} \log ^{3} \frac{1}{\beta}+O\left(\log ^{2} \frac{1}{\beta}\right)
$$


and

$$
\sum_{n=1}^{\infty} \frac{\log n}{n} e^{-n \beta}=O\left(\log ^{2} \frac{1}{\beta}\right)
$$

3. Proof of Theorem 1. Throughout the rest of the paper we assume $K$ is not totally real. The results and methods for $K$ totally real are exactly the same as for $K$ not totally real.

Now we have, for say $\sigma \geq 0$, and some constant $C>0$,

$$
\psi(s)=O\left(|t|^{C}\right) .
$$

This follows easily from the functional equation and an application of the PhragménLindelöf Theorem.

Now we consider the integral

$$
\frac{1}{2 \pi i} \int_{2-i \infty}^{2+i \infty} \Gamma(s) \psi(s) z^{-s} d s=\sum_{n=1}^{\infty} \frac{a(n)}{2 \pi i} \int_{2-i \infty}^{2+i \infty} \Gamma(s)(n z)^{-s} d s=\sum_{n=1}^{\infty} a(n) e^{-n z} \quad(\operatorname{Re} z>0) .
$$

Moving the line of integration to $\sigma=\alpha(0<\alpha<1)$ we get

$$
\frac{1}{2 \pi i} \int_{\alpha-i \infty}^{\alpha+i \infty} \Gamma(s) \psi(s) z^{-s} d s=\sum_{n=1}^{\infty} a(n) e^{-n z}=\phi_{0}(z),
$$

say. Hence, as in $[4$, p. 137], we have

$$
\int_{0}^{\infty}\left|\psi\left(\frac{1}{2}+i t\right)\right|^{2} e^{-2 \delta t} d t=\int_{0}^{\infty}\left|\phi_{0}\left(i x e^{-i \delta}\right)\right|^{2} d x+O(1)
$$

for sufficiently small $\delta>0$.

Now we remark that

$$
\phi_{0}\left(\frac{1}{i x e^{-i \delta}}\right)=\frac{2 \pi}{\sqrt{D}} i x e^{-i \delta} \phi_{0}\left(\frac{4 \pi^{2}}{D} i x e^{-i \delta}\right) .
$$

This transformation formula may be proven as in [4, p. 142], using the functional equation.

Now, as in [2, pp. 125-126], we have

$$
\begin{aligned}
\int_{0}^{2 \pi / \sqrt{D}}\left|\phi_{0}\left(i x e^{-i \delta}\right)\right|^{2} d x & =\int_{\sqrt{D} /(2 \pi)}^{\infty}\left|\phi_{0}\left(\frac{i}{x} e^{-i \delta}\right)\right|^{2} \frac{d x}{x^{2}} \\
& =\int_{\sqrt{D} /(2 \pi)}^{\infty}\left|\phi_{0}\left(\frac{1}{i x e^{-i \delta}}\right)\right|^{2} \frac{d x}{x^{2}} \\
& =\frac{4 \pi^{2}}{D} \int_{\sqrt{D} /(2 \pi)}^{\infty}\left|\phi_{0}\left(\frac{4 \pi^{2}}{D} i x e^{-i \delta}\right)\right|^{2} d x \\
& =\int_{2 \pi / \sqrt{D}}^{\infty}\left|\phi_{0}\left(i x e^{-i \delta}\right)\right|^{2} d x
\end{aligned}
$$


Now

$$
\begin{aligned}
\int_{2 \pi / \sqrt{D}}^{\infty}\left|\phi_{0}\left(i x e^{-i \delta}\right)\right|^{2} d x \\
=\int_{2 \pi / \sqrt{D}}^{\infty} \sum_{n=1}^{\infty} a(n) \sum_{m=1}^{\infty} a(m) e^{-n\left(i x e^{-i \delta}\right)} e^{-m\left(-i x e^{i \delta}\right)} d x \\
=\sum_{n=1}^{\infty} \sum_{m=1}^{\infty} \frac{a(n) a(m)}{i n e^{-i \delta}-i m e^{i \delta}} e^{2 \pi\left(-i n e^{-i \delta}+i m e^{i \delta}\right) / \sqrt{D}} \\
=\frac{1}{2 \sin \delta} \sum_{n=1}^{\infty} \frac{a^{2}(n)}{n} e^{-4 \pi n \sin \delta / \sqrt{D}} \\
\quad+2 \sum_{m=2}^{\infty} a(m) \sum_{n=1}^{m-1} a(n) \frac{(m+n) \sin \delta \cos [2 \pi(m-n) \cos \delta / \sqrt{D}]}{(m+n)^{2} \sin ^{2} \delta+(m-n)^{2} \cos { }^{2} \delta} e^{-2 \pi(m+n) \sin \delta / \sqrt{D}} \\
\quad-2 \sum_{m=2}^{\infty} a(m) \sum_{n=1}^{m-1} a(n) \frac{(m-n) \cos \delta \sin ^{2}[2 \pi(m-n) \cos \delta / \sqrt{D}]}{(m+n)^{2} \sin ^{2} \delta+(m-n)^{2} \cos ^{2} \delta} e^{-2 \pi(m+n) \sin \delta / \sqrt{D}} \\
=A_{1}(\delta)+2 A_{2}(\delta)-2 A_{3}(\delta),
\end{aligned}
$$

say. By Lemma 4,

$$
A_{1}(\delta)=\frac{A}{2 \delta} \log \left(\frac{1}{\delta}\right)+O\left(\frac{1}{\delta}\right)
$$

Also $A_{2}(\delta)$ may be evaluated, as in $[4$, p. 145], to give

$$
A_{2}(\delta)=O\left(\frac{1}{\delta}\right)
$$

The sum $A_{3}(\delta)$ is slightly more complicated, and may be evaluated as in [3, p. 150] to give

Collecting these extimates, we obtain

$$
A_{3}(\delta)=O\left(\frac{1}{\delta}\right)
$$

$$
\int_{2 \pi / \sqrt{D}}^{\infty}\left|\phi_{0}\left(i x e^{-i \delta}\right)\right|^{2} d x=\frac{A}{2 \delta} \log \frac{1}{\delta}+O\left(\frac{1}{\delta}\right),
$$

and this gives

$$
\int_{0}^{\infty}\left|\psi\left(\frac{1}{2}+i t\right)\right|^{2} e^{-\delta t} d t=\frac{2 A}{\delta} \log \frac{1}{\delta}+O\left(\frac{1}{\delta}\right) .
$$

Corollary 1 now follows from the theorem of $[4, p .136]$.

4. Proof of Theorem 2. We consider the integral

$$
\frac{1}{2 \pi i} \int_{2-i \infty}^{2+i \infty} \Gamma(s) \psi^{\prime}(s) z^{-s} d s=\sum_{n=1}^{\infty} \frac{b(n)}{2 \pi i} \int_{2-i \infty}^{2+i \infty} \Gamma(s)(n z)^{-s} d s=\sum_{n=1}^{\infty} b(n) e^{-n z} \quad(\operatorname{Re} z>0)
$$


Again, moving the line of integration to $\sigma=\alpha(0<\alpha<1)$, we get

$$
\frac{1}{2 \pi i} \int_{\alpha-i \infty}^{\alpha+i \infty} \Gamma(s) \psi^{\prime}(s) z^{-s} d s=\sum_{n=1}^{\infty} b(n) e^{-n z}=\phi_{1}(z)
$$

say.

We remark that

$$
\begin{aligned}
\phi_{1}\left(\frac{1}{i x e^{-i \delta}}\right)= & {\left[i x e^{-i \delta}\right]\left[\frac{4 \pi}{\sqrt{D}}\right]\left[\frac{1}{2} \phi_{1}\left(\frac{4 \pi^{2}}{D} i x e^{-i \delta}\right)+\log \left(\frac{\sqrt{D}}{2 \pi}\right) \phi_{0}\left(\frac{4 \pi^{2}}{D} i x e^{-i \delta}\right)\right.} \\
& \left.-\log x \phi_{0}\left(\frac{4 \pi^{2}}{D} i x e^{-i \delta}\right)+i \delta \phi_{0}\left(\frac{4 \pi^{2}}{D} i x e^{-i \delta}\right)\right]+O\left(x^{\alpha}\right) .
\end{aligned}
$$

This transformation formula may be proven using the functional equation, as in the first part.

Now, as in the first part,

$$
\begin{aligned}
& \int_{0}^{2 \pi / \sqrt{\mathrm{D}}\left|\phi_{1}\left(i x e^{-i \delta}\right)\right|^{2} d x=} \int_{\sqrt{\mathrm{D} /(2 \pi)}}^{\infty} \mid \phi_{1}\left(i x e^{-i \delta}\right)-2 \log (2 \pi / \sqrt{D}) \phi_{0}\left(i x e^{-i \delta}\right) \\
&-2 \log x \phi_{0}\left(i x e^{-i \delta}\right)+2 i \delta \phi_{0}\left(i x e^{-i \delta}\right)+\left.O\left(x^{\alpha-1}\right)\right|^{2} d x \\
&= \int_{\sqrt{D} /(2 \pi)}^{\infty}\left|\phi_{1}\left(i x e^{-i \delta}\right)\right|^{2} d x \\
&-2 \int_{\sqrt{D} /(2 \pi)}^{\infty} \log x \phi_{1}\left(i x e^{-i \delta}\right) \phi_{0}\left(-i x e^{i \delta}\right) d x \\
&-2 \int_{\sqrt{D} /(2 \pi)}^{\infty} \log x \phi_{1}\left(-i x e^{i \delta}\right) \phi_{0}\left(i x e^{-i \delta}\right) d x \\
&+4 \int_{\sqrt{D} /(2 \pi)}^{\infty} \log x\left|\phi_{0}\left(i x e^{-i \delta}\right)\right|^{2} d x+O\left(\frac{1}{\delta} \log ^{2} \frac{1}{\delta}\right) \\
&= \int_{2 \pi / \sqrt{D}}^{\infty} \sum_{n=1}^{\infty} \sum_{m=1}^{\infty} b(n) b(m) e^{-n\left(i x e^{-i \delta)}\right.} e^{-m\left(-i x e^{i \delta}\right)} d x \\
&-2 \int_{2 \pi / \sqrt{D}}^{\infty} \log x \sum_{n=1}^{\infty} \sum_{m=1}^{\infty} b(n) a(m) e^{-n\left(-i x e^{i \delta}\right)} e^{-m\left(i x e^{-i \delta}\right)} d x \\
&-2 \int_{2 \pi / \sqrt{D}}^{\infty} \log x \sum_{n=1}^{\infty} \sum_{m=1}^{\infty} b(n) a(m) e^{-n\left(i x e^{-i \delta}\right)} e^{-m\left(-i x e^{i \delta}\right)} d x \\
&+4 \int_{2 \pi / \sqrt{D}}^{\infty} \log x \sum_{n=1}^{\infty} \sum_{m=1}^{\infty} a(n) a(m) e^{-n\left(i x e^{-i \delta}\right)} e^{-m\left(-i x e^{i \delta}\right)} d x \\
&+O\left(\frac{1}{\delta} \log \frac{1}{\delta}\right) .
\end{aligned}
$$


Now let us look at the terms with $n=m$ in the above sum. They equal

$$
\sum_{n=1}^{\infty} a^{2}(n) \int_{2 \pi / \sqrt{D}}^{\infty} \log ^{2}\left(n x^{2}\right) e^{-2 n x \sin \delta} d x .
$$

This last sum equals, upon an integration by parts,

$$
\begin{gathered}
\frac{1}{2 \sin \delta} \sum_{n=1}^{\infty} \frac{\log ^{2}\left(4 \pi^{2} n / D\right) a^{2}(n)}{n} e^{-4 \pi n \sin \delta / \sqrt{D}}+\sum_{n=1}^{\infty} \frac{a^{2}(n)}{2 n \sin \delta} \int_{2 \pi / \sqrt{D}}^{\infty} \frac{4 \log \left(n x^{2}\right)}{x} e^{-2 n x \sin \delta} d x, \\
=B_{1}(\delta)+B_{2}(\delta), \text { say. }
\end{gathered}
$$

By Lemma 4,

$$
B_{1}(\delta)=\frac{A}{6 \delta} \log ^{3} \frac{1}{\delta}+O\left(\log ^{2} \frac{1}{\delta}\right) .
$$

Substituting $3 n x \sqrt{D} /(2 \pi)$ for $x$, we find

$$
\begin{aligned}
B_{2}(\delta)= & \sum_{n=1}^{\infty} \frac{4 a^{2}(n)}{n \sin \delta} \int_{3 n}^{\infty} \frac{\log x}{x} e^{-4 \pi x \sin \delta /(3 \sqrt{D})} d x \\
& -\sum_{n=1}^{\infty} \frac{2 a^{2}(n)}{n \sin \delta} \int_{3 n}^{\infty} \frac{\log n}{x} e^{-4 \pi x \sin \delta /(3 \sqrt{\mathrm{D}})} d x \\
& -\sum_{n=1}^{\infty} \frac{2 a^{2}(n)}{n \sin \delta} \int_{3 n}^{\infty} \frac{\log \left(9 D /\left(4 \pi^{2}\right)\right)}{x} e^{-4 \pi x \sin \delta /(3 \sqrt{D})} d x \\
= & B_{21}(\delta)-B_{22}(\delta)-B_{23}(\delta),
\end{aligned}
$$

say.

Now by Lemma 2, we find

$$
B_{21}(\delta)=\sum_{n=1}^{\infty} \frac{4 a^{2}(n)}{n \sin \delta} \sum_{m=3 n}^{\infty} \frac{\log m}{m} e^{-4 \pi m \sin \delta /(3 \sqrt{D})}+O\left(\sum_{n=1}^{\infty} \frac{a^{2}(n) \log n}{n^{2} \sin \delta} e^{-4 \pi n \sin \delta / \sqrt{D}}\right) .
$$

By interchanging the order of summation, we obtain

$$
B_{21}(\delta)=\frac{4}{\sin \delta} \sum_{m=3}^{\infty} \frac{\log m}{m} e^{-4 \pi m \sin \delta /(3 \sqrt{D})} \sum_{n=1}^{[m / 3]} \frac{a^{2}(n)}{n}+O\left(\sum_{n=1}^{\infty} \frac{a^{2}(n) \log n}{n^{2} \sin \delta} e^{-4 \pi n \sin \delta / \sqrt{D}}\right) .
$$

By Lemmas 3, 4, and 5,

$$
B_{21}(\delta)=\frac{4 A}{3 \delta} \log ^{3} \frac{1}{\delta}+O\left(\frac{1}{\delta} \log ^{2} \frac{1}{\delta}\right) .
$$

Similarly, by Lemmas 3, 4, and 5 ,

$$
\begin{aligned}
& B_{22}(\delta)=\frac{A}{3 \delta} \log ^{3} \frac{1}{\delta}+O\left(\frac{1}{\delta} \log ^{2} \frac{1}{\delta}\right), \\
& B_{23}(\delta)=O\left(\frac{1}{\delta} \log ^{2} \frac{1}{\delta}\right) .
\end{aligned}
$$


Thus

$$
B_{1}(\delta)+B_{2}(\delta)=\frac{7 A}{6 \delta} \log ^{3} \frac{1}{\delta}+O\left(\frac{1}{\delta} \log ^{2} \frac{1}{\delta}\right) .
$$

We now consider the terms with $n \neq m$, which are

$$
\begin{aligned}
& \int_{2 \pi / \sqrt{D}}^{\infty} \sum_{\substack{n=1 \\
n \neq m}}^{\infty} \sum_{m=1}^{\infty} b(n) b(m) e^{-n\left(i x e^{-i 8}\right)} e^{-m\left(-i x e^{i 8}\right)} d x \\
& -2 \int_{2 \pi / \sqrt{D}}^{\infty} \log x \sum_{n=1}^{\infty} \sum_{\substack{m=m \\
n \neq m}}^{\infty} b(n) a(m) e^{-n\left(-i x e^{i \delta}\right)} e^{-m\left(i x e^{-i \delta}\right)} d x \\
& -2 \int_{2 \pi / \sqrt{D}}^{\infty} \log x \sum_{\substack{n=1 \\
n \neq m}}^{\infty} \sum_{\substack{m=1 \\
\infty}}^{\infty} b(n) a(m) e^{-n\left(i x e^{-i s}\right)} e^{-m\left(-i x e^{i s}\right)} d x \\
& +4 \int_{2 \pi / \sqrt{D}}^{\infty} \log ^{2} x \sum_{n=1}^{\infty} \sum_{\substack{m=1 \\
n \neq m}}^{\infty} a(n) a(m) e^{-n\left(i x e^{-i \delta)}\right.} e^{-m\left(-i x e^{i \delta}\right)} d x \\
& =C_{1}(\delta)-2 C_{2}(\delta)-2 C_{3}(\delta)+4 C_{4}(\delta) \text {, }
\end{aligned}
$$

say.

Let us first consider $C_{4}(\delta)$.

$$
C_{4}(\delta)=\sum_{n=1}^{\infty} \sum_{\substack{m=1 \\ n \neq m}}^{\infty} a(n) a(m) \int_{2 \pi / \sqrt{D}}^{\infty} \log ^{2} x e^{-x[(m+n) \sin \delta+i(n-m) \cos \delta]} d x .
$$

Upon an integration by parts,

$$
\begin{aligned}
C_{4}(\delta)= & \sum_{n=1}^{\infty} \sum_{\substack{m=1 \\
n \neq m}}^{\infty} \frac{a(n) a(m)}{(m+n) \sin \delta+i(n-m) \cos \delta} \log ^{2}\left(\frac{2 \pi}{\sqrt{D}}\right) e^{-2 \pi[(m+n) \sin \delta+i(n-m) \cos \delta] / \sqrt{D}} \\
& +2 \sum_{\substack{n=1 \\
n \neq m}}^{\infty} \sum_{\substack{m=1 \\
n \neq m}}^{\infty} \frac{a(n) a(m)}{(m+n) \sin \delta+i(n-m) \cos \delta} \int_{2 \pi / \sqrt{D}}^{\infty} \frac{\log x}{x} e^{-x(m+n) \sin \delta} e^{i(-x)(n-m) \cos \delta} d x \\
= & C_{41}(\delta)+2 C_{42}(\delta),
\end{aligned}
$$

say.

We consider first, $C_{42}(\delta)$.

$$
\begin{aligned}
C_{42}(\delta)= & \sum_{\substack{n=1 \\
n \neq m}}^{\infty} \sum_{\substack{m=1 \\
\infty}}^{\infty} \frac{a(n) a(m)(m+n) \sin \delta}{(m+n)^{2} \sin ^{2} \delta+(n-m)^{2} \cos ^{2} \delta} \int_{2 \pi / \sqrt{D}}^{\infty} \frac{\log x}{x} e^{-x(m+n) \sin \delta} e^{i(-x)(n-m) \cos \delta} d x \\
& -\sum_{\substack{n=1 \\
n \neq m}}^{\infty} \sum_{\substack{m=1 \\
n \neq m}}^{\infty} \frac{i a(n) a(m)(n-m) \cos \delta}{(m+n)^{2} \sin ^{2} \delta+(n-m)^{2} \cos ^{2} \delta} \int_{2 \pi / \sqrt{D}}^{\infty} \frac{\log x}{x} e^{-x(m+n) \sin \delta} e^{i(-x)(n-m) \cos \delta} d x \\
= & C_{421}(\delta)-C_{422}(\delta),
\end{aligned}
$$

say. 
$C_{421}(\delta)$

$$
\begin{aligned}
= & \sum_{m=2}^{\infty} \sum_{n=1}^{m-1} \frac{a(n) a(m)(m+n) \sin \delta}{(m+n)^{2} \sin ^{2} \delta+(n-m)^{2} \cos ^{2} \delta} \int_{2 \pi / \sqrt{D}}^{\infty} \frac{\log x}{x} e^{-x(m+n) \sin \delta} e^{i(-x)(n-m) \cos \delta} d x \\
& +\sum_{n=2}^{\infty} \sum_{m=1}^{n-1} \frac{a(n) a(m)(m+n) \sin \delta}{(m+n)^{2} \sin ^{2} \delta+(n-m)^{2} \cos ^{2} \delta} \int_{2 \pi / \sqrt{D}}^{\infty} \frac{\log x}{x} e^{-x(n+m) \sin \delta} e^{i(-x)(n-m) \cos \delta} d x \\
= & C_{4211}(\delta)+C_{4212}(\delta),
\end{aligned}
$$

say.

$C_{4211}(\delta)=O\left(\sum_{m=2}^{\infty} \sum_{n=1}^{m-1} \frac{|a(n)||a(m)| 2 m \sin \delta}{(n-m)^{2} \cos ^{2} \delta}\left|\int_{2 \pi / \sqrt{D}}^{\infty} \frac{\log x}{x} e^{-x(m+n) \sin \delta} e^{i(-x)(n-m) \cos \delta} d x\right|\right)$ and upon substituting $e x \sqrt{D} /(2 \pi)$ for $x$, we obtain

$$
C_{4211}(\delta)
$$

$$
\begin{aligned}
= & O\left(\sum_{m=2}^{\infty} \sum_{n=1}^{m-1} \frac{|a(n)||a(m)| 2 m \sin \delta}{(n-m)^{2} \cos ^{2} \delta}\left|\int_{e}^{\infty} \frac{\log x}{x} e^{-2 \pi x(m+n) \sin \delta /(e \sqrt{D})} e^{i(-2 \pi x)(n-m) \cos \delta(e \sqrt{D})} d x\right|\right. \\
& +O\left(\sum_{m=2}^{\infty} \sum_{n=1}^{m-1} \frac{|a(n)||a(m)| 2 m \sin \delta}{(n-m)^{2} \cos ^{2} \delta}\left|\int_{e}^{\infty} \frac{1}{x} e^{-2 \pi x(m+n) \sin \delta /(e \sqrt{D})} e^{i(-2 \pi x)(n-m) \cos \delta /(e \sqrt{D})} d x\right|^{\prime},\right. \\
= & O\left(\sum_{m=2}^{\infty} \sum_{n=1}^{m-1} \frac{|a(n)||a(m)| 2 m \sin \delta}{(n-m)^{2} \cos ^{2} \delta} \frac{e^{-2 \pi(m+n) \sin \delta / \sqrt{D}}}{(m-n) \cos \delta}\right),
\end{aligned}
$$

by Lemma 1 . This sum may be evaluated as in [4, p. 145] to give

$$
C_{4211}(\delta)=O\left(\frac{1}{\delta}\right)
$$

Similarly $C_{4212}(\delta)=O\left(\frac{1}{\delta}\right)$, and so $C_{421}(\delta)=O\left(\frac{1}{\delta}\right)$.

By the same procedure as above, we find $C_{422}(\delta)=O\left(\frac{1}{\delta}\right)$, and so $C_{42}(\delta)=O\left(\frac{1}{\delta}\right)$.

We now consider $C_{41}(\delta)$.

$$
\begin{aligned}
C_{41}(\delta)= & \sum_{\substack{n=1 \\
n \neq m}}^{\infty} \sum_{\substack{m=1 \\
n \neq m}}^{\infty} \frac{a(n) a(m)(m+n) \sin \delta \cos [2 \pi(n-m) \cos \delta / \sqrt{D}]}{(m+n)^{2} \sin ^{2} \delta+(m-n)^{2} \cos ^{2} \delta} e^{-2 \pi(m+n) \sin \delta / \sqrt{D}} \\
& +\sum_{\substack{n=1 \\
n \neq m}}^{\infty} \sum_{\substack{m=1 \\
n}}^{\infty} \frac{a(n) a(m)(m-n) \cos \delta \sin [2 \pi(n-m) \cos \delta / \sqrt{D}]}{(m+n)^{2} \sin ^{2} \delta+(m-n)^{2} \cos ^{2} \delta} e^{-2 \pi(m+n) \sin \delta / \sqrt{D}} \\
= & C_{411}(\delta)+C_{412}(\delta),
\end{aligned}
$$

say.

$C_{411}(\delta)$ may be evaluated as in $[4$, p. 145$]$, to give

$$
C_{411}(\delta)=O\left(\frac{1}{\delta}\right)
$$


The second sum, again, is slightly more complicated and may be evaluated as in [3, p. 150] to give

$$
C_{412}(\delta)=O\left(\frac{1}{\delta}\right)
$$

Thus $C_{41}(\delta)=O\left(\frac{1}{\delta}\right)$, and so $C_{4}(\delta)=O\left(\frac{1}{\delta}\right)$.

Proceeding as above, we find similarly

$$
\begin{aligned}
& C_{1}(\delta)=O\left(\frac{1}{\delta} \log ^{2} \frac{1}{\delta}\right), \\
& C_{2}(\delta)=O\left(\frac{1}{\delta} \log \frac{1}{\delta}\right), \\
& C_{3}(\delta)=O\left(\frac{1}{\delta} \log \frac{1}{\delta}\right) .
\end{aligned}
$$

Collecting all the estimates, we obtain

$$
\int_{0}^{2 \pi / \sqrt{D}}\left|\phi_{1}\left(i x e^{-i \delta}\right)\right|^{2} d x=\frac{7 A}{6 \delta} \log ^{3} \frac{1}{\delta}+O\left(\frac{1}{\delta} \log ^{2} \frac{1}{\delta}\right) .
$$

Now $\int_{2 \pi / \sqrt{D}}^{\infty}\left|\phi_{1}\left(i x e^{-i \delta}\right)\right|^{2} d x$ may be evaluated as before to give

$$
\begin{aligned}
\int_{2 \pi / \sqrt{D}}^{\infty}\left|\phi_{1}\left(i x e^{-i \delta}\right)\right|^{2} d x & =\frac{1}{2 \sin \delta} \sum_{n=1}^{\infty} \frac{b^{2}(n)}{n} e^{-4 \pi n \sin \delta / \sqrt{D}}+O\left(\frac{1}{\delta} \log ^{2} \frac{1}{\delta}\right) \\
& =\frac{A}{6 \delta} \log ^{3} \frac{1}{\delta}+O\left(\frac{1}{\delta} \log ^{2} \frac{1}{\delta}\right) .
\end{aligned}
$$

Thus

$$
\int_{0}^{\infty}\left|\phi_{1}\left(i x e^{-i \delta}\right)\right|^{2} d x=\frac{4 A}{3 \delta} \log ^{3} \frac{1}{\delta}+O\left(\frac{1}{\delta} \log ^{2} \frac{1}{\delta}\right)
$$

and this gives

$$
\int_{0}^{\infty}\left|\psi^{\prime}\left(\frac{1}{2}+i t\right)\right|^{2} e^{-\delta t} d t=\frac{8 A}{3 \delta} \log ^{3} \frac{1}{\delta}+O\left(\frac{1}{\delta} \log ^{2} \frac{1}{\delta}\right) .
$$

Corollary 2 now follows as before.

\section{REFERENCES}

1. P. Barrucand, Quelques propriétés des coefficients des séries $L$ associées aux corps cubiques, C.R. Acad. Paris Sci. Sér. A-B 273 (1971), A960-A963.

2. Y. Motohashi, A note on the mean value of the Dedekind zeta-function of the quadratic field, Math. Ann. 188 (1970), 123-127. 
3. E. C. Titchmarsh, The mean-value of the zeta-function on the critical line, Proc. London Math. Soc. (2) 27 (1928), 137-150.

4. E. C. Titchmarsh, The theory of the Riemann zeta-function (Oxford: Clarendon Press, 1951).

5. L. Weinstein, The mean value of the derivative of the Dedekind zeta-function of a real quadratic field, Mathematika 24 (1977), 226-236.

Department of Mathematics

Present address:

BOSTON UNIVERSITY

Department of Mathematics

Boston, MassachusetTs 02215

TEMPLE UNIVERSITY

Philadelphia, Pennsylvania 19122 